\title{
RKKY-LIKE BIQUADRATIC EXCHANGE IN MAGNETIC SUPERLATTICES
}

\author{
W. Gruhn, Z. BąK and R. JaRoszewicz \\ Institute of Physics, Pedagogical University
}

Al. Armii Krajowej 13/15, 42-201 Częstochowa, Poland

\begin{abstract}
We show that in magnetic metallic superlattices along with the bilinear RKKY-reminiscent interaction between magnetic superlattices there can be an important quadrupole-quadrupole coupling. In an analytical way we derive the range functions of the quadrupol-quadrupole interaction and discuss its relation to magnetoresistivity.

PACS numbers: 75.70.Cn, 75.70.Fr
\end{abstract}

Since the discovery of antiferromagnetic coupling of ferromagnetic layers across a nonmagnetic metallic spacer layer, the magnetic superlattices (SL) have become an object of intense interest of both theoretical as well as experimental studies. The problem of RKKY-reminiscent spin polarization oscillations, with a long period and giant interface magnetoresistance attracted the most attention. The recently discovered, $\pi / 2$ coupling between magnetizations of the neighbouring magnetic layers [1] added new interest to the problem. This type of ordering suggests that along with the bilinear coupling of the type $J_{1} m_{1} \cdot m_{2}$ between magnetizations of adjacent layers there is an additional multipolar interaction. There were many attempts describing the mechanisms that give rise to biquadratic exchange, being a specific form of coupling between magnetic quadrupole moments of the ions (for details see Refs. [2, 3] and references therein).

The aim of the paper is to study a new intrinsic mechanism that originates in multipolar exchange coupling. In the following we will show that the quadrupole-quadrupole coupling can arise in a quite natural way from direct scattering of conduction electrons on magnetic multipole moments in the manner similar to bilinear RKKY interaction. The starting point for any description of metallic magnetic systems is the case of dilute alloys, when a few TM or RE ions are immersed in the sea of conduction electrons. The coupling of the ionic spin $\mathrm{S}_{n}$ with the itinerant electron spin $\sigma$ is usually taken as the contact interaction [4]: $H_{\mathrm{ex}}=-2 J S_{n} \sigma \delta(r)$. In the case of non-s magnetic ion state along with the scattering due to dipolar contact interaction there appears also scattering of conduction electrons on quadrupolar moments. Kondo [5] has proved that the interaction $\mathrm{V}_{\mathrm{qc}}(k)$ between conducting electrons and the quadrupoles is given by

$$
V_{\mathrm{qc}}=\frac{D}{k_{\mathrm{F}}^{2}} \sum_{k k_{1} \sigma \sigma_{1}}\left[\left(S_{n} \cdot k\right)\left(S_{n} \cdot k_{1}\right)-\frac{1}{3} S(S+1) k \cdot k_{1}\right] c_{k \sigma}^{+} c_{k_{1} \sigma_{1}}
$$


where $\sigma, k, \sigma_{1}$, and $k_{1}$ denote the spin and wave vector of the impact and scattered electron, respectively. $D$ is a constant that determines the strength of the scattering potential. Let us consider the quadrupole-quadrupole interaction mediated through the conduction electrons scattered on the ionic quadrupole moments according to Eq. (1). Since we are interested in the metallic system with superlattice (multilayer) symmetry we must account explicitly for the anisotropy associated with the growth direction. The wave function of conduction electrons in the layered system with modulation along the $z$-axis has the following general form [6]:

$$
\Psi_{K}(R)=\frac{1}{\sqrt{A}} \exp (\mathrm{i} k \rho) \Phi_{\kappa}(z),
$$

where we have used the notation $R=(\rho, z), \rho=\rho(x, y), K=(k, \kappa) . A-$ is the area of the region to which the motion of the electrons is confined. In the case of a SL consisting of thin magnetic, isolating layers separated by the nonmagnetic metallic spacers the single particle spectrum of our system is given by [6]

$$
E(k, \kappa)=\frac{\hbar^{2} k^{2}}{2 m}+2 T \cos (\kappa a),
$$

where $T$ is the hopping integral and $a$ denotes the SL modulation period. It is evident that Eq. (3) describes spectrum of mobile charge carriers also in the case when both constituents of SL are metallic. Assuming that the electron is described by the Bloch functions $\psi_{K}$ given by Eq. (2) the matrix element for the scattering from electron state $K$ to state $K_{1}$ by the ionic quadrupole moment is given by

$$
\begin{aligned}
V_{\alpha \beta}^{K K_{1}} & =\sum_{\alpha \beta} D\left[S_{i}^{\alpha} S_{i}^{\beta} n^{\alpha} n_{1}^{\beta}-\frac{1}{3} S(S+1) n^{\alpha} n_{1}^{\beta} \delta_{\alpha \beta}\right] \exp \left[\mathrm{i}\left(\boldsymbol{K}-\boldsymbol{K}_{1}\right) \cdot \boldsymbol{R}_{i}\right] \\
& =\sum_{\alpha \beta} \Delta_{\alpha \beta}^{K K_{1}} Q_{\alpha}^{\beta} \exp \left[\mathrm{i}\left(\boldsymbol{K}-\boldsymbol{K}_{1}\right) \cdot \boldsymbol{R}_{i}\right],
\end{aligned}
$$

where $S_{n}^{\alpha}$ and $n^{\alpha}$ denote the $x, y$ and $z$ components of the spin operator $S_{n}$ and the unit vector $n=K /|K|$ while $Q_{\alpha}^{\beta}$ denote the respective components of ionic quadrupole moment

$$
Q_{\alpha}^{\beta}=\left[S_{n}^{\alpha} S_{n}^{\beta}-\frac{1}{3} S(S+1) \delta_{\alpha \beta}\right] .
$$

Interaction (1) manifests itself in anisotropy of magnetoresistance [7], i.e. the resistivity $\rho_{H}$ is different according to whether the quadrupole axes are parallel or perpendicular to the current direction. As it has been shown [8] the anisotropy of resistivity is given by

$$
\frac{\rho_{\|}(H)-\rho_{\perp}(H)}{\rho_{0}}=D\left[\left\langle\left(S^{z}\right)^{2}\right\rangle-\frac{1}{3} S(S+1)\right]=D Q,
$$

where $\langle\ldots\rangle$ and $Q$ denote the thermal canonical average and quadrupolar ordering parameter, respectively. In the bulk systems, using formulae (1-6) one can determine the quadrupole term of localized moment-conduction electron interaction [7]. Since for some multilayered systems a giant magnetoresistance is observed one would expect that the interaction (1) is relatively strong and dominates other mechanisms of biquadratic exchange. 
Let us assume that the interaction between itinerant and localized moments is dominated by the potential given by Eq. (1). Similarly as in the case of dipolar contact scattering leading to RKKY coupling there should appear indirect coupling between quadrupole moments mediated via conduction electrons. Let us follow the perturbation procedure. Assuming that all conduction electron states below the Fermi level $\varepsilon_{F}$ are filled for both spin states the interaction between two quadrupole moments is mediated via double scattering $\left(K \rightarrow K^{\prime} \rightarrow K\right)$ with $\varepsilon_{K}<\varepsilon_{\mathrm{F}}$ and $\varepsilon_{K^{\prime}}>\varepsilon_{\mathrm{F}}$. The occupied $K$ states fill in $K=(k, \kappa)$ space the region limited by the conditions $\kappa \in\left(-q_{0}, q_{0}\right)$ ( $q_{0}$ is the wave vector that describes the SL period) and $k^{2} \leq k_{\mathrm{F}}(\kappa)$, where $k_{\mathrm{F}}$ in view of $\mathrm{Eq}$. (2) is given by

$$
k_{\mathrm{F}}=\left[2 m\left(\varepsilon_{\mathrm{F}}-2 T \cos (\kappa a)\right) / \hbar^{2}\right]^{1 / 2} \text {. }
$$

Under above-mentioned assumptions the interaction between quadrupole moments of magnetic ions $\left(Q_{i}\right.$ and $\left.Q_{j}\right)$ is given by [4]

$$
H\left(R_{i j}\right)=-\sum_{\alpha \beta \gamma \delta} Q_{\alpha}^{\beta} Q_{\gamma}^{\delta} \Lambda_{\alpha \beta}^{\gamma \delta}\left(\left|R_{i}-R_{j}\right|\right)
$$

where $\Lambda_{\alpha \beta}^{\gamma \delta}\left(\left|R_{i}-R_{j}\right|\right)$ is the range function of RKKY-like quadrupole-quadrupole interaction [4, 9], located at lattice sites $\boldsymbol{R}_{i}$ and $\boldsymbol{R}_{j}$, respectively

$$
\begin{gathered}
\Lambda_{\alpha \beta}^{\gamma \delta}\left(\left|R_{i}-R_{j}\right|\right)=\int_{-q_{0}}^{q_{0}} \frac{\mathrm{d} \kappa}{2 \pi} \int_{0}^{k_{\mathrm{F}}} \frac{\mathrm{d}^{2} k}{4 \pi^{2}} \int_{-q_{0}}^{q_{0}} \frac{\mathrm{d} \kappa^{\prime}}{2 \pi} \int_{k_{\mathrm{F}}}^{\infty} \frac{\mathrm{d}^{2} k^{\prime}}{4 \pi^{2}} \times \Delta_{\alpha \beta}^{K K^{\prime}} \Delta_{\delta \gamma}^{K^{\prime} K} \\
\quad \exp \left[\mathrm{i}\left(k-k^{\prime}\right) \rho_{i j}\right] \exp \left[\mathrm{i}\left(\kappa-\kappa^{\prime}\right) z_{i j}\right] /\left[E\left(k^{\prime}, \kappa^{\prime}\right)-E(k, \kappa)\right],
\end{gathered}
$$

where in view of Eq. (4) $\Delta_{\alpha \beta}^{K K^{\prime}}$ is the matrix element for the scattering from the electron state $K$ to $K^{\prime}$ by the quadrupole component $\mathrm{Q}_{\beta}^{\alpha}$. It is impossible to perform the integrations in Eq. (9) in an analytical way. However, we can gain information about the spatial dependence of the exchange parameters $\Lambda$. First of all let us note that due to the symmetry of the integration region among many possible coupling parameters only these for which $\alpha=\gamma$ and $\beta=\delta$ are nonzero (i.e. $\Lambda_{\alpha \beta}^{\alpha \beta}\left(\left|R_{i}-R_{j}\right|\right) \neq 0$ ). Moreover, using the procedure derived in paper [9] we can gain information about the oscillation period in the case when the interacting quadrupoles are displaced along the line parallel to the growth direction of the SL. In this case we can prove that the range functions of the quadrupole-quadrupole coupling behave as

$$
\sum_{\alpha \beta} \Lambda_{\alpha \beta}^{\alpha \beta}\left(\left|R_{i}-R_{j}\right|\right)=\Lambda(z) \sin ^{2}\left(q_{0} z\right) \ln (\rho) .
$$

Thus, the indirect multipole interaction in SL behaves in a similar way as the RKKY-like bilinear interaction. This resembles the situation in bulk metallic systems, where the biquadratic exchange has the same range function as the RKKY one [10]. The interaction (8) favours ferroquadrupolar ordering of the magnetic quadrupoles that belong to adjacent magnetic layers, therefore this mechanism cannot explain why in some SL system there appears perpendicular ordering (antiferro-quadrupolar ordering). It is evident that in these systems another mechanism dominates, this is probable the mechanism associated with layer thickness fluctuations $[11,12]$.

Financial support from the Committee for Scientific Research is gratefully acknowledged. 


\section{References}

[1] B. Heinrich, J.F. Cochran, M. Kowalevski, J. Kirscher, Z. Celiniski, A.S. Arrot, K. Myrtle, Phys. Rev. B 44, 9348 (1991).

[2] J. Inoue, J. Magn. Magn. Mater. 136, 233 (1994).

[3] P. Bruno, Phys. Rev. B 52, 411 (1995).

[4] M.A. Ruderman, C. Kittel, Phys. Rev. 96, 99 (1954).

[5] J. Kondo, Progr. Theor. Phys. 27, 772 (1962).

[6] D. Grecu, Phys. Rev. B 8, 1958 (1973).

[7] M.J. Sablik, P.M. Levy, J. Appl. Phys. 49, 2171 (1973).

[8] A. Friedrich, A. Fert, Phys. Rev. Lett. 33, 1214 (1977).

[9] Z. Bąk, W. Gruhn, J. Magn. Magn. Mater. 131, 210 (1994).

[10] H.H. Teitelbaum, P.M. Levy, Phys. Rev. B 14, 3058 (1976).

[11] J.C. Słonczewski, Phys. Rev. Lett. 67, 3172 (1991).

[12] R. Jaroszewicz, Z. Bąk, W. Gruhn, Acta Phys. Pol. A 89, 421 (1996). 